\title{
The Influence of the Body Image Presented Through TikTok Trend-Videos and Its Possible Reasons
}

\author{
Jiayan Liu ${ }^{1}$

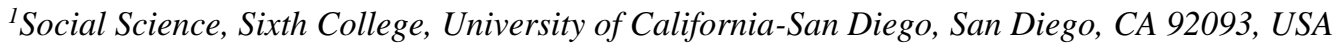 \\ Corresponding author's e-mail: ShiLiShuang@cas-harbour.org
}

\begin{abstract}
There are papers criticizing the impact of negative body imaging caused by social media. TikTok is an extraordinary Internet platform where everyone can upload videos to provide the public with the easiest way to get a sense of presence. TikTok makes the images under the beautiful filter tend to be standardized. The main purpose of this article is to discuss how this global popular social platform TikTok enhances body stereotypes and body humiliation trends, and what impact it has on the public and society. The research objects of this article are TikTok users and believers who fall into the trend of comments and compromise themselves in order to attract the attention of others and the typical videos they upload. In the main text, the author introduces and analyzes a series of popular TikTok videos with hash tags. The paper proves that TikTok does spread the trend of physical shame, and has a negative impact on the public's physical and mental health, body concepts, and potential threats to society.
\end{abstract}

Keywords: TikTok, negative body imaging, harmful effects, stereotyped, trend

\section{INTRODUCTION}

Nowadays, the media plays a vital role in guiding the values of young people. Among all values, body imaging is one of the most stereotyped in the public mindset. According to Gallivan's research [1], TV commercials, shows and movies are almost exclusively slender and fitlooking people. At present, the frequency of such images on social media is also particularly high. Studies have shown that girls who regularly use social media are 6 times more likely to engage in unhealthy weight control behaviors. Boys are four times more likely [1]. It can be seen that this situation has caused widespread body anxiety and may cause health problems for the public.

Therefore, this article will take the content and loyal users of the popular social software TikTok as the research objects to explore the human shame phenomenon reflected by the social platform. In addition, the author will analyze videos made by TikTok users that agree that people should treat unreal bodies as goals. Then through user reviews combined with research in psychology and sociology, the author will explore how social media can be an accomplice of physical shame, and analyze the negative effects of such a situation.
As the media theorist Postman [2] said, media will cleverly influence the environment and ultimately define and change our time. No one can predict when this humiliation trend will disappear, nor can it finally determine the public's view of human imaging. This article will provide a reference for how the public should view the body image on social platforms, and provide suggestions for the establishment of a healthier Internet social platform.

\section{TIKTOK USERS' OPINIONS ON BODY SHAMING}

TikTok has become a place for young people to express themselves in various ways, from lip-sync videos to crazy dances. The culture and structure of the platform encourage users to imitate each other and participate in hot topics. According to reports of watching TikTok videos every day, social platforms are flooded with unrealistic ideal bodies, that is, what the bodies of men and women look like to make them attractive. "When I first downloaded TikTok, I saw a lot of very, very negative body image videos," quoted from Kaufman [3], an active TikTok body activist in 2020 .

TikTok users are accustomed to these unrealistic body image videos, and they seem to take this trend for granted. Taking Khattab [4]'s research results in TikTok 
as an example, most users and video shooters have stereotypes about the body of both sexes. Khattab selected the most popular trends in video with hashtag of these videos has begun to attract a series of videos in response to user:. \#KarmaisaBitch. According to Feldman's article [5], all videos are obout the change of the appearance: The protagonist of the video shows an unattractive appearance at the beginning, and then says "Oh, okay. Karma is a bitch", and transforms into a more fashionable person with trendy makeup and hair.

These videos show the stereotypes about beauty and therefore have some recurring features. First, the user intentionally displays simulated behavior changes, from the softness in the first scene to the confidence and sexy in the second scene. Therefore, sexy is related to beauty and openness. In addition, medical conditions ranging from acne to implied more serious diseases are considered unattractive. Therefore, some abilities will be hinted to a certain extent in this process. The most obvious finding is that in some video samples, both female and male users pretended to be overweight in the initial scene, thus suggesting a sense of physical shame many times.

\#KarmaisaBitch is the subject of the biggest challenges has 145 million followers, while other alternatives (such as \#KarmaisaBitchChallenge) it has 4 million followers. On this short video platform, this seems to be a common scenario, and most users and video viewers do not think that physical shame is abnormal.

Instead, their "likes" and comments provide strong support for this appearance. Although most people cannot meet the so-called "slim" and "beautiful" standards, the crowd still recognizes and considers themselves "overweight". When these opinions continue to appear in the comment section of the short film, it is not difficult to find that most TikTok users desire a "perfect body" and have almost no fitness awareness. The comment section of TikTok videos can host annoying content, while users despise their bodies. Such comments can be considered "fat talk", which may lead to negative views of body image, which was first proposed by Mills \& Tyszkiewicz F. [6], a researcher at the School of Psychology at Deakin University in Australia.

In addition to the opinion that most people can humiliate the body, some TikTok stars have struggled with voices and comments criticizing their bodies over the years. However, this operation will not make any changes. Take a popular trending video \#DontJudgeMeChallenge with hashtags as an example. This video is composed of user-made videos that highlight facial blemishes such as acne or scars, and clearly add irony through makeup, only on camera Remove it on top to reveal a cleaner skin tone. Commented by Lin Shi[7], the movement is sometimes criticized for self-deception and spreading allegedly targeted elements that humiliate the body.

Then, the video did not fully reach the level of the title. Although the title of this challenge is more like a request for the public to reject the judgment, the structure of the video accepts or even seeks the judgment. It only requires viewers to postpone their judgment until the user changes its appearance to become more acceptable in the normalized aesthetic. However, without requiring any judgment, the video will cause judgment. Even some people want to eliminate this prejudice, which seems to enhance the value judgment based on human images.

\section{THE POTENTIAL THREATS OF NEGATIVE BODY IMAGING TO SOCIETY ENFORCED BY TIKTOK}

\subsection{Stereotyped discrimination, bullying, job loses}

Therefore, the stereotyped visual structure of the human body makes an important contribution to the hierarchical binary value judgment system of good and bad human images. In Engeln-Maddox's book [8], the media proposed traditional beauty ideals with a special preference for flawless facial features, skin tone, hair and figure. A relatively early study by Groesz, Levine and Murnen [9] confirmed that the slim figure presented in the media has an important influence on the public's satisfaction with their body.

As the trend changes, the thin body image has evolved into a healthy body image, but the single aesthetic of the body has not changed. For example, this has been emphasized in Tiggmann and Zaccardo [10]'s research on the hashtag \#fitspiration, which is a portrayal of the words "fit" and "inspiration". In addition, in another study on the self-customization of female body images in Instagram, Fardouly, Willburger, and Vartanian [11] discussed the role of adaptability in defining hard-to-obtain body images.

"This kind of fixation will lead to the marginalization of aging and disabled people." proposed by Tiidenberg and Gómez Cruz [12]. "Even when appearance is irrelevant, they tend to focus only on women's appearance and physical characteristics rather than personality characteristics or abilities. This trend is very common. Both men and women tend to build women's value mainly through the following methods: Studies have shown that their bodies look very beautiful." [13].

If the public is biased against body image, then there may be more incidents of physical discrimination and invisible bullying in schools and workplaces. These harmful problems will definitely cause confusion in our society's value judgment system. The new digital media TikTok can attract people's attention through its 
attractive features (for example, tags and influencers), thereby accelerating this stereotype.functions such as hashtags and influencers who aspire for amount of attention.

\subsection{Gender stereotypes}

Empirical evidence confirms that many women report using models displayed by the media as comparison objects when assessing their appearance. Ringrose et al. [14] stated that the digitally mediated value judgements associated with these videos ascribe to a normative heterosexualised performances of feminine and masculine desirability nowadays. Such heterosuxalised context resulted in normalizing the sexualization of the female body as Evans, Riley and Shankar [15] explained in their book. Similarly, Siibak [16] mentioned that men present their body image as sexualized and romantic objects, influenced by stereotypical visual representation of masculinity on social media. In his study of constructing masculinity in social networks, Siibak argues that posing techniques by users in social networks are influenced by advertising trends. This is evident in the videos in the TikTok videos discussed before for both men and women.

Encompassing around the stereotypical visual representation of extreme sexualization every day, netizens of our era are confronting a big challenge. Putting an overemphasized-clear line between the appearance of two sex is quite unfriendly to the transgender, the LGBTQ, etc.—- the minority.

\subsection{Younger generation, future of society}

According to Gallivan's presentation [1], on a typical day American children ages 8-18 are engaged with some form of media for 7.5 hours. It's easy to think that TikTok can probably be one of these media for children to play and watch.

Dina Borzekowski, professor at Johns Hopkins school of public health notes: "Social media may have a stronger impact on children's body image than traditional media. Messages and images are more targeted: if the message comes from a friend it is perceived as more meaningful and credible." She also emphasizes that children most at risk are those with more exposure to media messages and less exposure to rational, clear messages from supportive adults.

In order to catch up the trends, millions of children could try dangerous things like this, not to say about taking the harmful body criteria for granted. There are so many worries about the value judgement system of children. The article written by Porterfield [17] discussed that taking some essential action to the videos about negative body image and eating disorders is especially important for TikTok, with roughly 18 million of the platform's daily users believed to be under the age of 15 .

\section{REASONS WHY TIKTOK IS CAPABLE OF INFLUENCING NETIZENS' OPINION ON BODY IMAGING}

The first reason is the algorithm. The algorithm used by TikTok can track what users like to browse and interact with, and then recommend similar content to them to increase the number of views on the platform. In addition, TikTok also has communities divided by interest, which not only cultivates users' sense of belonging, but also increases competitive pressure. Thanks to this feature, TikTok has attracted millions of viewers and followers. According to the website data report [18], there are approximately 689 million monthly active users.

In addition, users can also promote their ideal ways to lose weight through the recent trend on the platform, including fitness, diet and fitness clothing. According to Dempster, A. [19], this rising trend has attracted the attention of people who are prone to eating disorders and body imaging disorders. Many people began to question their consumption, their dress and their figure. Because their fitness habits, eating habits, and dressing habits did not match the images presented in these popular videos, they began to wonder if they were lazy, undisciplined or failed people. These videos take advantage of others' anxiety about life and dissatisfaction with the present. Many people think that if they live like these Internet celebrities, their lives will be as good as theirs. As a result, these comparisons make people think "I should eat better", "Why do I not look like this?" or "I wish I could do this." Therefore, the decline in shame and self-worth will destroy the thinking and behavior of those who are greatly affected. However, as E. Lantos [20] said, it is important to note that these advertised standards are not suitable for everyone's own body shapes and needs.

In addition, from the Internet perspective, the group also showed similar body image and diet problems. This phenomenon is sometimes referred to as peer infection, and was first proposed by Hutchinson, Lapi, and Paxton. [twenty one].

What is more, it has been found that appearancerelated comments from friends strengthen the relationship between physical dissatisfaction and eating disorders [22]. Therefore, peers have been shown to be an important source of affecting young people's body image and eating problems.

\section{CONCLUSION}

In conclusion, this article proves that the emerging new media TikTok has a significant impact on the value judgment system that guides people, causing serious 
social problems. In order to solve these problems, people should pay attention to the algorithm and the cliques on the platform. It is worth noting that TikTok is not isolated. It also links its shareability with major social networks (such as Facebook, Twitter and Instagram) to gain more access. The widespread promotion of short video platforms has a huge social impact. However, this influence may not proceed smoothly due to some criticisms or even legal battles over its content. In order to jointly build a new media environment in a healthier way in the future, scholars, listeners and software developers should first understand the consequences of the content published on the platform. Second, users need to consciously collect different opinions to avoid the negative impact of information cocoons.

\section{REFERENCES}

[1] H.R. Gallivan. Presentation about teens, Social Media And Body Image, thousands of lives restored, healing eating disorder. Park Nicollet Melrose Center. 2014. pp:1-3.https://www.macmh.org/wpcontent/uploads/2014/05/18_Gallivan_Teenssocial-media-body-image-presentation-HGallivan-Spring-2014.pdf

[2] N. Postman. Amusing ourselves to death. 1985. The Medium Is the Metaphor.

[3] S. Kaufman. Young women on how TikTok has warped their body image during COVID pandemic. NBC News. 2020. https://7news.com.au/lifestyle/healthwellbeing/not-worth-it-young-women-on-howtiktok-has-warped-their-body-image--c-1179929

[4] M. Khattab. Synching and performing: body (re)presentation in the short video app TikTok. Osuva Open Science. 2020. http://widerscreen.fi/numerot/2019-1-2/synchingand-performing-body-re-presentation-in-the-shortvideo-app-tiktok/

[5] B. Feldman. "Karma's a Bitch" is the Rare Meme Combining Riverdale and Kreayshawn. New York Magazine.

2018. http://nymag.com/intelligencer/2018/01/what-isthe-karmas-a-bitch-meme.html

[6] T.F. Mills. Fat Talk and Body Image Disturbance: A Systematic Review and Meta-Analysis. Psychology of Women Quarterly. 2016. https://journals.sagepub.com/doi/abs/10.1177/0361 684316675317

[7] J. Linshi. Here's How the "Don't Judge Challenge" Totally Backfired. Time. 2015. http://time.com/3948968/dont-judge-challenge/.
[8] R. Engeln-Maddox. Buying a Beauty Standard or Dreaming of a New Life? Expectations Associated with Media Ideals. Psychology of Women Quarterly. 2006, 30(3): 258-266. Doi: 10.1111/j.14716402.2006.00294.x.

[9] L.M. Groesz, P. Michael. Levine and Sarah K. Murnen. The Effect of Experimental Presentation of Thin Media Images on Body Satisfaction: A Meta analytic Review. International Journal of Eating Disorders. 2002, 31(1): 1-16. Doi: 10.1002/eat.10005.

[10] M. Tiggemann and M. Zaccardo. "Strong Is the New Skinny": A Content Analysis of \#fitspiration Images on Instagram. Journal of Health Psychology. 2018, 23(8): 1003-1011. Doi: $10.1177 / 1359105316639436$.

[11] J. Fardouly, B.K. Willburger, and L.R Vartanian. Instagram Use and Young Women's Body Image Concerns and Self-Objectification: Testing Mediational Pathways. New Media \& Society. 2018, 20(4):

1380-1395.

Doi:10.1177/1461444817694499.

[12] K. Tiidenberg and E.G. Cruz. Selfies, Image and the Re-Making of the Body. Body \& Society. 2015, 21(4): 77-102. Doi:10.1177/1357034X15592465.

[13] N. Ellemers. Looks Do Matter, Especially for Women, and Also at Work. Psychology Today. 2018.

https://www.psychologytoday.com/us/blog/socialclimates/201809/looks-do-matter-especiallywomen-and-also-work

[14] J. Ringrose, L. Harvey, R. Gill and S. Livingstone. Teen Girls, Sexual Double Standards and 'Sexting' : Gendered Value in Digital Image Exchange. Feminist Theory. 2013, 14(3): 305-323. Doi: $10.1177 / 1464700113499853$.

[15] A. Evans, S. Riley and A. Shankar. Technologies of Sexiness: Theorizing Women's Engagement in the Sexualization of Culture. Feminism \& Psychology, 2010, 20(1): 114-131. Doi: $10.1177 / 0959353509351854$.

[16] A. Siibak. Constructing Masculinity on a Social Networking Site: The Case-study of Visual SelfPresentations of Young Men on the Profile Images of SNS Rate. YOUNG: Nordic Journal of Youth Research. 2010, 18(4): 403-425. Doi: $10.1177 / 110330881001800403$.

[17] C. Porterfield. TikTok Takes On 'Body Shaming' With A Ban on Ads for Fasting Apps. Forbes. 2020.https://www.forbes.com/sites/carlieporterfiel 
d/2020/09/23/tiktok-takes-on-body-shaming-witha-ban-on-ads-for-fasting-apps/?sh=7f3859a9389e

[18] Global Social Media Overview. DATAREPORTAL. 2020. https://datareportal.com/social-media-users

[19] A. Dempster. Tik Tok weight loss videos fueling eating disorders amid coronavirus lockdowns, health experts say. 2020. ABC news. https://www.abc.net.au/news/2020-05-13/expertsconcerned-tiktok-content-fuelling-eatingdisorders/12215986

[20] E. Lantos. Tik Tok: Fears videos may trigger eating disorders. BBC wales news. 2020. https://www.bbc.com/news/uk-wales-52919914

[21] D.M. Hutchinson \& R.M. Rapee. Do friends share similar body image and eating problems? The role of social networks and peer influences in early adolescence. Behaviour Research and Therapy, 2007, 45(7), 1557-1577. Doi:10.1016/j.brat.2006.11.007.

[22] K.J. Forney, L.A. Holland \& P.K. Keel. Influence of peer context on the relationship between body dissatisfaction and eating pathology in women and men. International Journal of Eating Disorders, 2012, 45(8), 982-989. Doi:10.1002/eat.22039. 